(Darmkatarrh) neuerdings wieder umfangreichere Verwendung gefunden. Hierher gehürt auch das Boluphen, ein Gemisch von Bolus, Phenol und Formaldehyd, ein brauchbares Antiseptikum für Tiere.

Die Silberpräparate werden trotz ihrer hohen Desinfektionskrait heute in der Veterinärmedizin wegen des unerschwinglichen Valutapreises noch kaum benutzt. Vor dem Kriege war das Silbernitrat als Ätzmittel für Wunden mit schwacher Granulation sehr beliebt, zumal die Epithelisierung größerer Wundflächen unter dem Silberschorfe günstig beeinflußst wurde. $\mathrm{Ob}$ es als innerliches Antiseptikum einen Wert besitzt, muß wegen seiner leichten Zersetzlichkeit bezweifelt werden. Hierfur wird vielmehr das kolloidale Silber (Kollargol, Elektrargol und ähnliche Präparate, die meist intravenüs einverleibt werden), benutzt, dessen therapeutische Wirkung aber bei den Infektionskrankheiten (Druse, Petechialfieber, Kälberruhr, Maul- und Klauenseuche, Sepsis) sicher überschätzt wird. Die modernen Silberpräparate, Protargol (Silbereiweiß), Tannargan (Tanninsilbereiweiß), Albarg in, I ch th arg a n (Ichthyolsilberverbindung), das Actol (milchsaures Silber), Itrol (zitronensaures Silber) usw. haben den Vorzug vor dem salpetersauren Silber, daß sie nicht eiweißfällend wirken, durch Chlorionen nicht selbst gefüllt werden und daher bei der innerlichen Anwendung erhebliche Vorteile aufweisen. Trotzdem sind sie als Heilmittel für Haustiere nicht verwendbar, da zu teuer.

Von den Quecksilberverbindungen stehen, wie bereits erwähnt, das Sublimat als Wunddesinfizienz und bei der Großdesinfektion sowie das KalomeI als Darmdesinfizienz und Laxans an erster Stelle, doch ist die Anwendung derselben beim Rind kontraindiziert wegen der überaus großen Empfindlichkeit gegen Quecksilber (akuter Mercurialismus). Die neueren organischen $Q$ uecksilberpräparate, die in der Humanmedizin bei der Behandlung der Syphilis in Anwendung kommen, haben keine Aufnahme als Tierheilmittel gefunden. $\mathrm{Ob}$ das Merkaffin (Quecksilberpurinverbindung), dem man bei der Hundestaupe spezifische Heilwirkung zuschreibt, den Anpreisungen entsprechen wird, ist recht fraglich.

Als sehr mildes Antiseptikum wird die Borsäure in der Augenheilkunde auch bei Tieren mehr als irgendein anderes Mittel angewendet, da sie infolge ihrer schwachen Dissoziation kaum reizend wirkt und relativ ungiftig ist. Daher spielte sie mit ihrem Natrium salz (Borax) ebenso wie die Salizylsäure als Konservierungsmittel für Fleisch- und Wurstwaren, Konserven und Milch eine große Rolle, bevor die Verwendung zu diesem Zwecke gesetzlich verboten wurde In der iiblichen Konzentration ist sie nicht imstande, die Bakterien der P'aratyphusgruppe (Fleischvergifter) abzutüten und nur durch die Unterdrieckung der Füulnis erhält sie die Nahrungsmittel scheinbar in einem einwandfreien Zustande. In grüßeren Mengen aufgenommen wirkt sie für den Menschen giftig. Die Perborate, die unter verschiedenen Namen angepriesen wurden und deren Wirkung auf der Bihdung von Wasserstoffsuperoxyd beruht, haben praktisch keine Bedeutung erlangt.

Die Beobachtung, daß auch organische Farbstoffe stark bakterizide Eigenschaften haben, hat zur Darstellung einer ganzen Anzah von l'räparaten geführt, die als Therapeutika für die Veterinärmedizin als Gewinn bezeichnet werden müssen. Ein Teil von ihnen wirk ganz spezifisch gegen gewisse Blutparasiten und soll daher später bei den Infektionskrankheiten Erwähnung finden. Hier sollen nur kurz die Mittel berücksichtigt werden, die sich in der Wundbehandlung bewilhrt haben. Zunächst ist es das Pyoktanin (Methylviolett), ein Gemenge von Penta- und Hexamethyl-p-Rosanilinchlorhydrat, das wegen seiner austrocknenden schorfbildenden Eigenschaften in der Tierleeilkunde umfangreich angewendet wird, insbesondere bei der Behandlung von großen durch Erosionen entstandenen Wundflachen, wie sie oft bei Pferden infolge Dekubitus, Quetschung, Verbrennung hervorgerufen werden. Trotz der lästigen Farbe hat das Methylviolett sich gegen das sogen. gelbe Pyoktanin (salzsaures Auramin) wegen seiner stïrkeren Desinfektionskraft behauptet. Die zahlreichen Konkurrenten (zum Teil ebenfalls in die Gruppe der Triphenylmethanreihe hineingehörend), 7. B. Malachitgrün, Phenylblau, Anilinblau, Fuchsin, Toluidinblau u. a., deren bakterizide Wirkung nicht abgeleugnet werden soll, sind entbehrlich. - Dagegen sind einige A zofarbstoffe, Amidoazotoluol-Azo- $\beta$-naphthol (Scharlachrot) und das Amidoazotoluol selbst, sowie dessen farbloses Acetylierungsprodukt (Pelidol, Azodermin) infolge ihrer günstigen Einwirkung auf Epithelzellen bekannt geworden und werden bei granulierenden Flächenwunden zwecks schnellerer Utberhäutung angewendet. Infolge ihrer relativ geringen desinfizierenden Eigenschaften wurde durch Kombination mit Jodolen (Jodeiweiß) ein verbessertes Präparat ( $\mathrm{zz}$ dolen) erzielt, das auch unter Tierärzten Freunde gefunden hat.

Ein großer Nachteil vieler unserer gebräuchlichsten Desinfektionsmittel ist die Unzulänglichkeit ihrer Wirkung auf die Mikroorganismen im lebenden Gewebe. Selbst stark wirkende Mittel wie das Sublimat büßen bei Gegenwart von Gewebseiweiß, das ja in jeder Wunde vorhanden ist, ganz erheblich von der Wirkung ein, die sie in vitro zu entfalten imstande sind. Daß sie außerdem vielfach zu starken Ätzungen der Wunde führen und durch die Zerstörung der lebenden Zelle vielen Erregern einen günstigen Nährboden verschaffen, ist bereits oben erwähnt worden.

Infolgedessen hat es auch nicht an Bestrebungen gefehlt, die eine Sterilisierung der Wunde nicht auf chemischem, sondern auf physikalischem Wege erreichen wollten und zwar durch Fixierung der Bakterien durch starke Klebekraft gewisser Harze. Eins dieser Präparate, das
Mastisol, eine Lösung von Mastixharz in Benzolrhloroformgemisch, hat in der Tierheilkunde festen Fuß gefaßt. Der große Vorteil, den die Mastisolmethode bietet, liegt nicht nur in einer schnellen Sterilisierung des Operationsfeldes durch die Arretierung der Erreger, sondern ganz bes inders in der Vereinfachung der Verbandstechnik. Die hohe Klebekraft, die durch Wasser- und Wundexsudate nicht beeinträchtigt wird, ermöglicht an jeder beliebigen Kürperstelle, wo es bisweilen unmüglich ist, einen Verband anzulegen, Wattebausch und Tupfer zu fixieren, wodurch die Sekundärinfektion verhindert und viel Verbandsmaterial gespart wird. Im Kriege hat sich der Mastisolverband bei Pferden außerordentlich bewährt.

(Fortsetzung folgt.)

\section{Die Vorgänge bei der Gerbung. Il.}

\author{
Von Dr. W. MOELLER.
}

(Nach einem am 2. Dezember 1921 im Hamburger Bezirksverein des Vereins deutscher Chemiker gehultenen Vortrag.)

(SchlnB von S. 154.)

In dem Schlußkapitel meiner Arbeit: „Die Elementaritruktur der Lederfaser" ${ }^{28}$ ) wies ich auf die großen Fortschritte bin, die durch die netre Methode der rontgenographischen Unter*uchung von Kristallen auch far die Faserstolf- und Lederchemie und Erforschung der Struktur der Lederfaser gegeben sind. Inzwischen ist diese von mehreren Seiten, besonders von Lehman ${ }^{20}$, angedeutete Moglichkeit der weileren Erforschung der Faserstoffe auf physikalischem Wege durch Rontgenstrahlen Tatsache geworden. Die neuere Literalur weist zahlreiche Untersuchungen nach dem L a ueschen Verfabren auf, und immer mohr scheint sich auch die von mir vertretene Anschauung 7. verbreiten, daß die Elementarteile der sogenannten amorphen Körper und somit auch der Haut- und Lederfaser entsprechend der v. Niigelischen Theorie kristallinische Struktur besitzen. Ich verweise auf die Arbeiten von Herzog und Jancke ${ }^{3 v}$ ) usw., die die verschiedenartigsten sogenannten amorphen Korper und Faserstoffe aach dem Rontgenverlahren untersucht haben. In zahlreichen Fällen $h_{\text {nt }}$ sich ergeben, besonders mit Bezug auf die Cellulosefuser, daß die Anordnung der Teilchen entsprechend einem einachsigen Kriglall vorhanden ist, und $\mathrm{d}_{\mathrm{n}} \mathrm{B}$ die einzelnen Teilchen kristallinische Struktur bositzen mulssen. Mit der Untersuchung der Haut- und Lederfaser im Röntgenlicht habe ich mich kürzlich ebenfalls beschäftigt ${ }^{* 2}$ ). Es war vorauszusehen, da $B$ die von mir in der Arbeit "Die Elementarstruktur der Lederfaser" ${ }^{32}$ ) gezogenen Schluffolgerungen, die zum Teil auf spekulativer, zum Teil aber auch auf experimenteller Grundlage gewonnen werden, ihre Bestatigung erfahren $u$ ürden. Die rðn'genspektrographischen Úntersuchungen der Abbauprodukte des Bindegewebes, der Gelatine, liegen ebenlalls in neebrfacher Rirhtung vor, doch ist uber die Deutung der Resultate keine endgultige Entscheidung getroffen. Die aufyentellte Behauptung über die anqebliche amorphe Beschaffenheit des Gelantinevels sind noch nicht als eindeutig zu betrachton, sondern es kann sich um ein Gemenge landeln, bei dem der eine Korper eine kristallinische Struktur zeigt und der mit einem anderen amorphen vermischt ist. Ich vertrat hekinntlich in zahlreichen Arbeiten die Anschauung, daß wir es bei der Gelatine-Gallerte mit einem netzartigen kristallartigen Gewebe zu tun haben, dessen Zwischenräume mit einer Micellarsubstauz ausgefüllt ist. Diese Auffassung ähnelt der kürılich von Zsigmond $\mathrm{y}^{33}$ ) gé̉ußerten Anschauung. Auch Polanji $i^{34}$ ) beschäPtigt sich mit der Faseruntersuchung im Röntgenlicht nacle einer etwas abgeänderten Methode, bei welcher diejenigen Subsłanzen, deren Untersuchung nach der bisherigen Methode der Köntgen-Spektrographie Schwierigkeiten bietet, besser erfaßt werden, indem unter sehr hohem Druck his zum Eintreten des Fließens eine Substanz erhalten wird, deren Teilchen eine nach der Hauptachse geordnele Struktur erhalten. Man ersieht daraus, $\mathrm{daB}$ auch die röntgenographisçenen Untersuchungsmethoden noch lange nicht erschøpft und auch fur die Lederforschung noch zahlreiche Anwendungsmöglichkeiten gegeben sind ${ }^{35}$ )

Die vorstehenden Zusammenhänge der Micellarhypothese mit denjenigen der Lederchemie beziehen sich zunälchst auf die strukturellen Verhältnisse der Lederfaser selbst. Der erweiteiten Anwendung der Micellarhypothese von v. Nïgeli, die ich in einer großßeren Arbeit im Zusammenhang mit der Theorie der Gerbung entwickelte ${ }^{36}$, liegt ebenfalls die Anschauung von v. Nägeli zugrunde. Hereits v. Nägeli hat eine sogenannte Umkleidungstheorie der Micellen entwickelt und spricht dabei von einem "Panzer“, infolge Anlugerung

28) L c. Collegium 1918, Nr. 584.

29) Ascher-Spiro, Ergebnisse d. Physiol, 16, 508 [1917]

so) Die Naturwissenschaften 1921, 320. - Es ist selbstverständlich, daB auf diesem eben erst erschlossenen Gebiete noch viele Widersprüche aufzuklären sind, wie diese kurze Arbeit der beiden Forscher zeigt. Ferner Ztschr. I. angew. Chemie 1821, Nr. 59, S. 385

so) Zischr. f. Leder u. Gerberui-Chemie 1, 41-47 [1921]

s1) Collegium 1913, Nr. 549-557.

32) Collegum 1918, Nr $577-534$

33) Kolloidchemis 1920, 408

31) Die Naturwissenscbatten 1921, 337

85) Vgl. auch den Artikel von Herzog and Jancke in der JubilfumsFestschrift der Kaiser-Wilhelm-Gesellschaft 1921.

s6) Collegium 1918, Nr. 577-584. Die Elementarstruktur der Lederfaser. 
fremder Molekule an diejenigen der Micellarverbïnde der Eiweißmicellen, wodurch eine Unangreifbarkeit gegenuber hydrolytisrhen und fermentativen Prozessen ohne weiteres gegeben ist ${ }^{37}$ ). Auch uber die Richtigkeit dieser Vorgänge lassen sich mit Hilfe physikalischer Methodun zablreiche Beweitmittel erbringen. Sprziell mit dem hydrolytischen and fermentativen dbban der Haut und Lederf iser habe ich mich in zahlreichen, neueren Arbeiten beschäftigt ${ }^{38}$ ) und man begegnet bei diesen Untersuchungen stets wieder den Zusammenhănyen, die zwischen den Vorgängen der Lederbildung und der Micellarhypothese v. Nagelis bestehen. - v. Nigeli hat diemit der Theorie der Gerbung zusammenhingenden Vorgange in seiner Micellarhypothese zweimal ganz klar ausgesprochen:

I. Vgl. Nageli und Schwendner, Das Mikroskop. Leipzig 1877, S. 424.

"Überdies kőnnen Micellen, ohne ibre Anordnung zu verändern, sich soweit voneinander entfernen, daß Molekule anderer Substanzen, zu denen sie die Affinilat besitzen, sich zwischen diese einschieben und eine formliche Hulle um sie bilden."

III. Vgl. Nägeli, Theorie der Gärung. Mümchen 18i9, S. 137 u. 138. ${ }_{n}$ Dieses einschichtige, beinahe unbewegliche Hätchen von Wassermolekülen um die Substanzmicelle ist nicht nur bei der Diosmose zu hertlcksichtigen. Seine Starrheit wird vermehrt du'ch die Einlagerung fremdartiger unorganischer und organischer Stoffe, welche keiner organisierten Substanz ganz mangeln und die wir uns wohl in keiner anderen Weise vorslellen können, als daß die Molekllle dieser Stoffe sich an die Micellen anlegen .... Durch die Einlagerungen wird, wenn sie in geringer Menge vorhanden sind, das Wachstum der Micelle innerhalb des Hilulcbens nicht gehemmt, wohl aber die Unlöslichkeit bedeutend vermehrt. Sind sie aber reichlicher vorhanden, so können sie gleichsam einen Panzer um die Micelle bil len und dieselbe nicht nur wachstumsunfälig, sondern aurh für Quellungs- und Lősungsmittel fast unangreifbar machen."

Wie man sieht, schließt die v. Nägelische Micellartheorie weder die chemische noch die kolloidchemische Theorie der Gerbung völlig aus. Auf alle Fille ist man aber gezwungen, zu einer Umkleidungstheorie der Micellen nach der einen oder anderen Richtung bei der Erklärung der Lederbildung auf Grund der Anschauungen von v. Nägeli zurlickzukehren.

Die Bestätigung dieser Anschauung glaubte ich in den Resultaten der ultramikroskopischen Untersuchungen der Haut- und Lederfaser zu finden. Ferner auch unter Nutzbarmachuag der eingangs erwihnten Methoden der rüntgen-spektrographischen Untersuchungen. Auch aus diesen letzteren Ergebnissen ließ sich die kristallinische Struktur der verschiedenen Lederfasern deutlich nachweisen.

Wiahrend nun nach der einen Seite hin die Erhaltung des Micellarverbandes für das Zustandekommen der Gerbung und die Beschaffenheit der daraus entstehenden Lederfaser erforderlich ist, laufen aber viele vcïbereitenden Maßnahmen mit der tierischen Haut.zur Überführung in Hautblüße auf einen teilweisen Abbau der Micellen hinaus, und zwar beim Äschern durch Alkali und beim Schwellen durch Säuren. Sowohl Alkali als auch Säure greifen das Kollagen mit der Zeit der Einwirkung in bedeutendem Maße an, indem neben der Schwellung und Quellung auch gleichzeitig eine Loslösung und Aufspaltung der die einzelnen Micellen zusammensetzenden Eiweißmolekulle eintritt, und man findet demnach in w in Berührung waren, schon einen geringen Anteil an $\mathrm{Hautsub-}$ stanz, aber in noch viel höherem Maße, wenn man die Hautsubstanz selbst mit sehr verdünnten Alkalien und Säuren behandelt. Ich stellte fest, daß jede HautblöBe an das Wasser eine ganzbestimmte Menge Hautsubstanz abgibt, und zwar ziemlich konstant, wenn die Hautsubstanz sterilisiert war. Man kann demnach von einer proteolytischen Konstante der Hautblüße gegenüber Wasser sprechen ${ }^{39}$. Bei Einwirkung von Siauren und Alkalien erhöht sich diese an die Lösung abgegebene Hautsubstanzmenge proportional der Zeit der Einwirkung und der Konzentration der angewandten Alkalien und Sz̈uren. Auch Salzlüsungen der verschiedensten Art greifen die Hautsubstanz in wüsserigen Lösungen in ganz bedeutendem Maße an, und diese Einwirkung oder Abbau der Eiweißsubstanzen ist in gewisser Beziehung deswegen von der elektrolytischen Dissoziation des verwendeten betreffenden Salzes abhängig. ${ }^{\prime}$ ). Das Wesen einer jeden Gerbung besteht nun darin, die sonst unter gewöhnlichen Bedingungen bei Einwirkung von Wasser oder feuchter atmosphärischer Luft in der tierischen Haut eintretende bakterielle, d. $h$. fermentative oder hydrolytische Zersetzung zum Stillstand zu bringen und diese fundamentale Eigenschaft besitzen tatsächlich alle wirklich gerbenden Systeme. Man kann demnach mit dem Begriff "gerbende Substan ${ }^{-}$alle solche Verbindungen bezeichnen, die die Eigenschaft haben, die hydrolytische und fermentative Zersetzung der Haut zum Stillstand zubringen und auf unbegrenzte Zeit zu verhindern. Irgendwelche GerbstoffIösung wird demnach bei der Einwirkung auf die Hautsubstanz den

${ }^{37}$ ) Theorie und Gärung, Müncben 1879, S. 138.

$\left.{ }^{38}\right)$ Die Beziehungen zwischen Hydrolyse und Adsorption. Collegium 1920, Nr. 599-604. Ztschr. 1. angew. Chemie 1920, I, 2?1-223.

39) Die Beziehungen zwischen Hydrolyse und Adsorption. Collegium 1920, $109,152,209,267$.

10) Zischr. t. Leder- u. Gerbereichemio 1, 12 [1921]. an und fur sich bestehenden geringen hydrolytischen Zustand, der nach meinen Untersuchungen etwa $1-2 \%$ der trockenen Hautsubstanz beträgt, nicht weiter erhöhen, sondern man findet in der Restbrühe nach der Einwirkung der gerbenden Lösung nicht mehr abgebaute Hautsubstanz, als die Haut vor der Einwirkung des Gerbmittels an das Wasser abgegeben hat ${ }^{* 1}$ ). Die Feststellung dieser Hautsubstanz in wässeriger Lüsung oder auch in der zurückbleibenden Gerbstofflüsung geschah auf sehr einfache Weise nach der Kjeldall-Methode, indem ein aliquoter Teil der verwendeten Restbrühe zur Trockne gebracht, und in der Trockensubstanz der Stickstoff naeh Kjeldahl bestimmt wird. Durch die Untersuchungen von v. Schröder und PaeBler ${ }^{4}$ ) ist bekannt. daß der Stickstoffgehalt verschiedener Hautblüßen an sich ziemlich konstant ist und bei der Rindsblüße etwa $17,8 \%$ betriggt, d. h. jedes Prozent an gefundenem Stickstoff ist etwa das 5,8 fache an Hautsubstanz. Schon durch frithere Untersuchungen von v. Schröder und PaeBler ${ }^{43}$ ist bekannt, daß die Hautsubstanzverluste beim vegetabilischen Gerbprozeß an sich sehr gering sind und im Durchschnitt nur etwa $1,5 \%$ der trockenen Hautsubstanz belragen. $Z u$ diesen Ziffern waren v. Schröder und $\mathrm{PaeBler}{ }^{44}$ ) auf indirektem Wege durch Bestimmung des Stickstoffgehaltes in der verwendeten trockenen Haut und Bestimmung des Stickstoffgehaltes in dem trockenem Leder nach der Fertigstellung gelangt. Die nach meiner Methode festgestellten Hautsubstanzverluste deckten sich in liberraschender Weise mit diesen von v. Schröder und Paeßler gefundenen Resultaten. In Wirklichkeit kann man aber, wie bereits oben bemerkt, von einem wirklichen Hautsubstanzverlust nicht sprechen, sondern diese $1,5 \%$ an gefundenen Abbauprodukten der Haut sind gleich von Anfang an nicht als intakte Hautsubstanz anzusprechen und der Gerbung auch nicht fahig. Zieht man demnach diesen proteolytischen Faktor der verwendeten BlüBe von den gefundenen proteolytischen Anteilen nachderGerbung ab, so wird die Differenz gleich Null. Man solltz nun meinen, daß diese Tatsache ganz selbstverständlich ist und kaum irgendeiner Bestätigung bedarf, da ja doch das Wesen der Gerbung gerade in der Erhaltung der Hautsubstanz in Form von Leder bestehen soll. Wie wenig aber gerade in dieser Richtung von einer selbstverständlichen Tatsache gesprochen werden kann, wurde von mir mit Hilfe der in dem letzten Jahrzehnt so vielfach in Anwendung kommenden neueren synthetischen Gerbstoffe nachgewiesen. Es wurde nämlich von $\mathrm{mir}$ durch umfangreiche Experimente nachgewiesen ${ }^{4}$ ), daß die synthetischen Gerbstoffe nach den versehiedensten Methoden hergestellt, in Form von sulfogruppenhaltigen organischen Verbindungen sich ganz anders als Gerbstoffe verhalten, und $z$ war genau wie jede andere Säure, $z$. B. freie Sehwefelsỉure, die die Haut proportional der Zeit und Konzentration angreifen und abbauen. Die Hautsubstanzverluste sind demnach mit solchen künstlichen Gerbstoffen ganz beträchtlich, und dementspreohend auch die Rendementsverluste dergewonnenen Leder; denn jedem Prozent Hautsubstanz entspricht etwa beim vegetabilischen Gerbverfahren mehr als das Doppelte an Ledersubstanz. Diese Erscheinung steht nun im krassen Widerspruch zu dem oben aufgestellten Gesetz der Konstanz der Hauptsubstanz bei jedem Gerbprozeß. Jedoch ist die Erklärung für diese Erscheinung einfach darin zu suchen, daß die bisherigen kunstlichen Gerbstoffe neben dem wirklich gerbenden System auch noch freie molekulardisperse Sulfosiduren enthalten, die stark elektrolytisch dissoziiert sind und die Hautfaser dementsprechend angreifen. Dieses Verhalten freier Sulfogruppen ktinstlicherGerbstoffe ist demnach eines von den eingangs bereits erwähnten Beweismitteln fur die absolute Notwendigkeit der Abwesenheit freier Wasserstoffionen bei jedem wirklichen Gerbvorgang. Sind freie Wasserstoffionen anwesend in Form von freien Säuren, wie etwa Schwefelsäure im Leder, die bekanntlich eine auBerordentlich zerstörende Wirkung selbst in stark verdünnten Lösungen ausübt, so findet ein allmählicher fortgesetzter Abbau der Hautsubstanz selbst noch in Form von Leder statt. Selbst unverhaltnismäßig minimale Spuren von solchen freien Säuren bewirken starke Zerstórung der Leder nach der Gerbung. Aus diesem Grunde müssen alle Theorien, die auf irgendwelche Ionenbetätigung bei dem Gerbprozeß hinauslaufen, abgelehnt werden, da durch solche Dissoziationsvorgänge gerade das Ümgekehrte bei der Hautsubstanz erreicht wird, als der $Z$ week der Gerbung ist, nämlich Abbau und Zerstörung der Hautsubstanz. Leder ist nach meiner Theorie tierische Haut, deren Elementarteilchen gegenüber fermentativen und hydrolytischen Einflüssen geschütrt sind. Ich Iasse dabei vollständig diejenige Frage offen, die seit Beginn der Gerbereichemie Gegenstand der theoretischen Erörterungen ist, nämlich die Form der Bindung zwischen Gerbstoff und Hautfaser oder wie dieser Schulz gegenüber den er-

41) Ledertechnische Rundschau 1820, Nr. 12, 13, 14, 15, 23, 24; 1921 ,

Nr. 1. 2, 8, 9, 11, 13

42) Gerbereichemie, Berlin 1908, 608 .

43) Gerbereichemie, Beılin 1908, 448.

4) Gerbereichemie, Berlin 1908, 448.

45) Das geibereichemische Verbalten der Sallograppe klustlicher Gerbstoffe, Collegium 1920, Nr. 607, S. 520. 
wähnten Einflüssen erreicht wird. Daß es sich aber hierbei nicht um eine einfache kolloide Ausflockung entgegengesetzt geladener Kolloide handeln kann, dafür sprechen viele Umstände, und auch gegen die rein chemische Auffassung des Gerbvorganges lassen sich vielerlei Momente anführen. Meine letzten Untersuchungen über diesen Gegenstand, die sich ebenfalls nach der hydrolytischen Seite hin bewegen, beschüftigen sich mit dem Verhalten der verschiedenen Lederarten gegenüber der Einwirkung des heißen Wassers ${ }^{46}$ ). Bekanntlich hat schon Fahrion ${ }^{47}$ ) im Jahre 1908 eine Methode der Lederprufung geschaffen, die darauf beruht, die bei der Einwirkung des heiBen Wassers aus dem Leder herausgelösten löslichen Substanzenzu bestimmen, und die zurllckbleibende Menge an Trockensubstanz, bezogen auf die angewendete Menge Ledersubstanz, ist die sogenannte Wasserbeständigkeitszahl. Hierbei geht Fahrion von der Annahme aus, dab die gelösten Substanzen aus Leim bestehen sollten, ohne allerdings hierfür den Nachweis zu führen. Bei dieser Methode schnitt das vegetabilische Leoer am allerschlechtesten ab und ergab die niedrigste Wasserbestiindigkeitszahl. Fahrion bezeichnete demnach diese Lederart als nach einer Art Pseudogerbung zustande gekommen. Im Gegensatz hierzu ergaben besonders die sämischgaren und chinongaren Leder außerordentlich hohe Wasserbeständigkeitszahlen. Fahrion untersuchte aber nicht näher die Beschaffenheit der aus dem Leder herausgelösten Substanzen, und es erschien mir daher notwendig, festzustellen, in welchem Maße die Hautsubstanz an diesen dureh das heiße Wasser herausgelösten Substanzen beteiligt ist. Die Ausführung dieser Methode war ebenso einfach, wie die voraufgegangene hydrolytische Bestimmungsmethode, indem eine aliquote Menge des Filtrates der Heißwasserprobe zur Trockne gebracht und darin Stickstoffbestimmungen ausgeführt wurden. Dabei ergab sich, daß die in dem vegetabilischen Leder vorhandene Hautsubstanz mindestens die gleiche, wenn nicht hohere Wasserbeständigkeitszahl zeigt, wie alle anderen Lederarten. Durchweg kann man sagen, daß annähernd die gleiche Menge Hautsubstanz bei allen echten Ledern durch das heiBe Wasser herausgelöst wird. Ein Unterschied besteht lediglich darin, daß neben dieser Hautsubstanz auch ein bestimmter Anteil des aufgenommenen Gerbmittels mit herausgelöst wurde, und in dieser Beziehung gibt das vegetabilische Leder am meisten Gerbstoff ab. Aber das ist ja auch ganz crklärlich, denn das vegetabilische Leder enthält ja auch ungleich größere Mengen an Gerbstoff, wie alle anderen Lederarten, und daß diese Gerbstoffe teilweise wasserlöslich sind, war ja eigentlich vorauszusehen. Man kann demnach keineswegs sagen, da $B$, wie : uf Grund der Fahrionschen Methode angenommen wurde, dieses oder jenes Leder viel oder wenig Hautsubstanz an das heiße Wasser abgibt, sondern alle Lederarten geben gleichmäßig wenig ab, und $z$ war etwa 9-10\% der in Leder vorhandenen Hautsubstanz. Das ist eine tuberraschende Tatsache, denn auch hier zeigt sich wiederum eine gewisse Konstanz, indem der Abbau der Hautsubstanz selbst in heißem Wasser durch die Gerbmittel gleichmäBig zum Stillstand gebracht wird, und man kann demnach von einem proteolytischen Faktor der im Leder vorhandenen Hautsubstanz gegenüber heißem Wasser sprechen. Die Rückstände, die nach dieser Methode erhalten wurden, stellen aber selbstverständlich keine Ledersubstanz mehr dar, denn die Hautfaser als solche wird durch die Einwirkung des heißen Wassers und durch die Fortfïhrung der Hydrolyse bis zu $10 \%$ der gesamten Hautsubstanz in Gelatine übergeführt, und letzten Endes bleibt als Rückstand eine in heißem Wasser gegerbte Gelatine als unlösliche Masse zurück. Daraus nuß logischerweise der Schluß gezogen werden, গåß auch schon gewöhnliche Gelatine als solche bei der Ausflockung oder Gerbung in wässeriger Lösung ungeführ die gleichen Ziffern an gegerbten Anteilen ergeben muß, wie die Hautfaser, und diese Voraussetzung bestätigte sich in glänzender Weise $\left.{ }^{\star 5}\right)$. Bei der Gerbung der Gelatine mit verschiedenen vegetabilischen und mineralischen Gerbmitteln zeigte sich, daß nur etwa $80-90 \%$ der Gelatinemasse an der Gerbung teilnahm, während der Rest als zu weit abgebaute Form der Gelatine im Filtrat in ungegerbter Form wiedergefunden wurde. In diesem $\mathrm{Zu}$ sammenhang mit der Heißwasserprobe wurden noch interessante Feststellungen und Parallelen gezogen in dem Verhalten der künstlichen Gerbstoffe nach dieser Methode in ähnlicher Weise, wie bei den hydrolytischen Versuchen in kalten wăsserigen Lösungen. Es ergab sich nämlich bei der gleichen Untersuchung ${ }^{49}$ ) der verschiedenen mit den im Handel befindlichen künstlichen Gerbstoffen mit Sulfogruppen gegerbten Ledern, daB diese eine auBerordentlich geringe Beständigkeit gegenüber heißem Wasser zeigten und da $\beta$ die in diesen Ledern befindliche Hautsubstanz nicht wie die wirklichen mittels vegetabilischer, mineralischer und sämischer Gerbung hergestellten Leder nur $10^{\circ} i_{11}$ an das Wasser abgeben, sondern ganz bedeutend mehr, zum Teil mehr als $50 \%$ der in den Ledern vorhandenen Hautsubstanz. Es ist natürlich Sache des Geschmacks, ob man diese Leder überhaupt noch als Leder bezeichnen will oder ob sie eine Zwischenstufe zwischen roher Haut und wirklichem Leder dar-

16) Zischr. f. Leder- u. Gerbereichemie 1, 47,[1921]

i) Chemiker-Zeitung 1908, Nr. 75. Collegium 1908, S. 495.

18) Ztschr. I. Leder- u. Gerbereichemie 1, 80 [1921].

18) Ztschr. f. Leder- u. Gerbereichemte 1, 100 [1921]. stellen. Auf alle Fille nehmen, wie durch diese beiden grundlegenden Versuche festgestellt, die mit den bisherigen künstlichen Gerbstoffen mit Sulfogruppen gegerbten Lederarten eine deutliche Sonderstellung ein. Auf diese fundamentalen, ab weichenden Eigenschaften sind auch die zahlreichen Mißerfolge bei der ganz bedeutenden Anwendungsform dieser Gerbstoffarten während der Kriegszeit zurückzuführen. Es ist natürlich Aufgabe der Gerbstoffchemie, die künstlichen Gerbstoffe nach dieser Richtung hin zu verbessern, und es wird zweifellos ein Leichtes sein, entweder durch Beseitigung dieser nachteiligen Eigenschaften oder durch Auffindung von anderen Richtungen für den Aufbau synthetischer Gerbstofftypen auf diesem Gebiete fortzuschreiten Die oben dargelegten Beziehungen zwischen dem hydrolytischen Abbau einerseits und dem verursachten Stillstand desselben durch die Gerbmittel andererseits lassen nun manche, bisher unbekannte Ursachen besonders in der Mineralgerbung und die zahlreichen Mißerfolge bei verschiedenen neueren Mineralgerbungen, besonders der Eisengerbung, ganz selbstverständlich erscheinen. Schon $\mathrm{Kn}$ a p ${ }^{5 u}$, der als der eigentliche Erfinder der Chromgerbung anzusprechen ist, hat vor mehr als 50 Jahren darauf hingewiesen, daß nur die vorsichtig mit Alkali neutralisierten Chrom- oder Eisensalze zu brauchbaren Resultaten führen können, während die nicht neutralisierten Gerbsalze, wie Chromalaun für sich und besonders die Eisensalze, stets ein brüchiges, blechiges Resultat ergeben. Über die Ursache dieser Erscheinung und den Zweck der Neutralisation wird noch heute vielfach gestritten. Über den einen Zweck sind sich allerdings so gut wie alle Chemiker im klaren, nämlich die Uberführung der Chromverbindungen in komplexe Salze. Aber das wesentlichste Moment, nämlich die Ausschaltung der elektrolytischen Dissoziation, wurde dabei nicht erkannt. Bei den von mir ausgeführten Untersuchungen ${ }^{51}$ ) ergab sich nun, daß bei Einwirkung von genau neutralisierten Chrom- und Eisenverbindungen in wässeriger Form auf die Hautsubstanz kein Abbau der Hautsubstanz eintrat, sondern eine wirkliche Gerbung, indem der proteolytische Faktor auch bei sehr langer Einwirkungsdauer erhelt 3n blieb. Im Gegensatz hierzu gaben aber nicht neutralisierter Chromalaun und Eisenlisungen schon nach ganz kurzer Zeit sehr beträchtliche Mengen Hautsubstanz an die Brühe $a b$, ein Zeichen, daß kein wirklicher Gerbvorgang vorlag sondern lediglich eine Wirkung der Wasserstoffionen der elektrolytisch dissoziierten Salze. Bei den Eisensalzen ergab sich aber selbst in neutralisiertem Zustande von einem gewissen Zeitpunkt ab noch die gleiche Erscheinung des Abbaues der Hautsubstanz, und dieser Umstand gibt uns auch eine Erklärung für die Tatsache, daß es bis heute noch kein einwandfreies Eisenleder gibt, welches, längere Zeit aufbewahrt, keine Zerstörungserscheinungen der Hautfasern zeigt. Es gibt zwar Kombinationsgerbungen bei der Eisengerbung, wobei solche Mittel hinzugefügt werden, die die zerstörende Wirkung der Eisensalze durch Aufnahme der freiwerdenden Wasserstoffionen verhindern; jedoch kann man solche Leder zunächst nicht als reine Eisenleder bezeichnen. Der Grund, weshalb sich die Eisensalze anders verhalten und deren Einführung in die Gerbereichemie immer noch Schwierigkeiten bereiten, ist deren auBerordentlich leichtes Dissoziationsvermügen in der Lősung und auch im Leder. Die bisherigen Eisengerbverfahren laufen demnach alle auf eine Kombination mit irgendeinem Mittel als Akzeptor für die freiwerdenden Wasserstoffionen hinaus, um dadurch den sehr leicht eintretenden Abbau der Hautsubstanz zu beseitigen. Man könnte nun versucht sein, eine gewisse Parallele zwischen diesen Mißerfolgen bei der Eisengerbung und den früheren Mißerfolgen mit saurem Chromalaun und den neueren künstlichen organischen Gerbmitteln zu ziehen, und tatsächlich liegt eine gewisse Ähnlichkeit zwischen diesen Gruppen vor. Erst, wenn es gelingt, diese Körperklasse in wässeriger Form in stabile, nicht dissoziierte Form uberzuführen; wird es möglich sein, dauernd praktisch brauchbare Resultate für alle Gerbzwecke und Lederarten zu erzielen.

Zum Schluß möchte ich kurz auf die sich aus diesen Darlegungen ergebenden Folgerungen für die Theorie der Gerbung eingehen, wofär als Ausgangspunkt wiederum die Definition des Gerbstoffes selbyt gewählt werden muB. Nach der von mir aufgestellten Theorie ${ }^{32}$ ) der Gerbstofflösungen muß der wesentlichste Bestand teil einer Gerbstoffverbindung das Vorhandensein eines für sich allein in Wasser absolut unloslichen Korpers oder die Bildung desselben bei Berührung mit der Hautsubstanz sein. Andererseits muß dieser Körper durch ein besonderes Verfahrender Peptisation, welches in der Kolloidchemie zahlrejche Verwendung findet, in eine kolloidlösliche Form übergefthrt werden. Bei den pflanzlicben Gerbstoffen finden diese Vorgänge ohne weiteres beim Auslaugen der Rinden und Hözer mittels heißem Wasser statt, und der sogenannte Peptisationsvorgang geht automatisch vor sich. Bei den übrigen Gerbarten, sei es mineralischer oder anderer Natur, wird der Peptisationszustand desabsolut unloslichen Körpers durch chemische Eingriffe hervorgebracht und zwar bei den Chrom-und Eisengerbsalzea durch Zusatz von Alkali zu den sauren Salzen. Derartige Systeme stellen

${ }^{50)}$ Natur und Wesen der Gerberei and des Leders, München 1858, S. $28 / 29$.

51) Die Mineralgerbungen IV, Collegium 1921, 67.

82) Die Peptisationserscheinungen in Gerbstofflösungen, Collegium 1915, 50. Die pllanzlichen Gerbstoffkolloide, Collegium 1915/16 Nr. 648-556. 
demnach eine Auflösung von Oxyden dieser Metalle in ihren sauren Salzen dar. Im allgemeinen wird angenommen, daB es sich hierbei in der Hauptsache um komplexe Salze handelt. Es sprechen aber viele Umstände dafür, daß außer diesen komplexen Verbindungen freies Chrom-oder Eisenoxyd in solchen Lösungengelöst wird, und nach meiner Theorie beruht ausschließlich a uf dem Vorhandensein in dieserkolloiden peptisierten freien Hydroxyde der Gerbeffekt dieser Salze ${ }^{53}$ ). Eine scheinbare Ausnabme bilden die bei der Sämisch-und Aldehydgerbung verwendeten Stoffe. Jedoch habe ich bei der Ald ehydgerbung einwandfrei nachgewiesen ${ }^{54}$ ), daß Aldehyd in wässeriger Lösung im Kontakt mit der Hautsubstanz sofort zur energischen Polymerisation und Kondensation neigt und demnach spontan diejenigen Substanzen erzielt werden, die die Unangreifbarkeit der Faser gegenüber hydrolytischen und fermentaliven Substanzen bewirken. Bei der normalen. Sämischgerbung liegt anscheinend eine vollständig $\mathbf{a b}$ weichende Gerbart, und demnach auch ein vollig abweichender Vorgang bei der Bindung der gerbenden Bestandteile mit der Hautfaser vor. Aber auch hier trifft die von mir aufgestellte Theorie ohne Einschränkung zu. Nach dem üblichen praktischen Sämischgerbverfahren wird zwar durch mechanische Bearbeitung der Haut mit dem Gerbmittel dieses mit der Hautfaser vereinigt. Das ist jedoch lediglich darauf zurückzuführen, daß man bis heute noch kein Verfahren kannte, Fettsubstanzen in eine solche kolloide peptisierte Form überzuführen, daß es, nach den üblichen Brühengerbverfahren verwendet, die Haut in Leder überführt. Ich habe aber nachgewiesen ${ }^{55}$, daß z. B. die gewöhnlichen Phenole vorzügliche Peptisatoren für alle bei der Sämischgerbung verwendeten Fettsubstanzen darstellen, und daß es auf diesem Wege möglich ist, die Haut in genau derselben Weise ohne mechanische Bearbeitung durchzugerben, wie bei jedem anderen Gerbverfahren. Bei allen Gerbvorgängen kann man demnach immer wieder dieselbe Beobachtung machen, daß alle gerbenden Substanzen a priori eine Widerstandsfähigkeit gegenüber hydrolytischen und fermentativen Einflüssen besitzen müssen, um diese Eigenschaft bei der Vereinigung mit der Hautfaser auf diese selbstzu übertragen und diese gegenüber den erwähnten Einflüssen zu schützen. Die Vorgänge, die sich bei der Übertragung dieser unlöslichen unangreifbaren gerbenden Substanzen auf die Hautfaser mitteilen, entsprechen im allgemeinen denjenigen Vorgängen, die man in der physikalischen Chemie mit "Adsorptionsvorgänge" bezeichnet. Über die Natur und das Wesen der Adsorption selbst und die Kräfte, die die Bindung $z$ wischen Adsorbenz und Adsorbentien bewirken, wird immer noch gestritten. Die chemische Richtung sieht in der Adsorptionskraft lediglich die Betätigung von Nebenvalenzen und versurht, unter Erweiterung der sngenannten Wernerschen Theorie der Komplexverbindungen die Adsorptionserscheinungen auf Valenzkräfte zurück7.uführen. Ist diese Theorie richtig, so müßten schließlich auch die sogenannten Adhäsionskräfte mit unter den Begriff der Adsorptionskräite fallen, und jede Oberflächenwirkung würde nichts anderes als Valenzbetätigung darstellen. Andererseits führt die kolloidchemische Theorie ${ }^{56}$ ) die Gerbvorgänge auf die Betätigung besonderer elektrischer Kräfte zurück, die mit den Valenzkräften in keinem Zusammenhang stehen und damit nicht vergleichbar sind. Für die vorliegende Gerbtheorie sind diese Fragen zunächst von sekundärer Bedeutung, denn die Adsorptionsvorgänge kommen nur für die Form der Hineinfuhrung der gerbenden Bestandteile in die Haut mit Hilfe des Peptisators, der den Adsorptionsgesetzen folgt, in Frage. Durch die Adsorption wird die Trennung des Peptisators von der neptisierten Substanz, und die Ausfällung oder das Niederschlagen der unangreifbaren unlöslichen peptisierten Substanzen auf die einzelnen Micellen des Micellarverbandes der Leder verursacht. Darin besteht nach meiner Auffassung das Wesen einer jeden Gerbung.

[A. 47.]

\section{Berichtigung.}

Der Name des Verfassers des Berichtes über die Chemie der synthetischen Süßstoffe (Ang. Chem. 35, 133 [1922] lautet: W. Herzog, nicht, wie infolge Druckfehler dort zu lesen ist, Herzrog. Des weiteren muß es auf S. 133, r. Sp. unten statt Kelcher: Kalcher heißen.

\section{Aus Vereinen und Versammlungen.}

Deutsche Landwirtschafts-Gesellschaft.

Die Wintertagung (90. Hauptversammlung) fand vom 13 . bis 18. Februar im Geschäftshaus der D. L. G. Berlin, Dessauer Str. 14 und in anderen Versammlungsräumen statt. Von den Verhandlungsgegenständen waren die folgenden von chemischem Interesse:

Geh. Reg.-Rat. Prof. Dr. Tacke, Vorsteher der Moor-Versuchsstation, Bremen, berichtete über ,Neuere Erfahrungen auf dem Gebiete der Moorkultur's. Besprochen wurde der Einfluß einer versehiedenen Entfernung der Röhrenstränge bei Dränungen von Moorböden

53) Die Mineralgerbung IV, Collegium 1921, 68.

54) Ztschr. \&. Leder- u. Gerbereichemie 1, 54

55) Collegium 1919,61.

58) Journ. Am. Leather Chem. Assoc. 1917, 76-80. und verschiedener Tiefe der Entwässerungsgräben, die zweckmäßige Art der Entwässerung bei Kultivierung versandeter Seebecken, Fragen der Bodenbearbeitung des Moorbodens, der Düngung, insbesondere der Produktionsleistung der Phosphorsäure auf Moorboden im Vergleich zu mineralischen Bodenarten, besondere Erfahrungen bei Verwendung verschiedener Kalisalze, insbesondere der schwefelsauren Kalimagnesia, Anbauversuche, die Ansaat neuanzulegender und die Nachsaat $\mathrm{zu}$ verbessernder Moorwiesen und Weiden, die Wirtschaftlichkeit einer Stickstoffdüngung auf Wiesen und Weiden. Besonders hervorgehoben wird die im letzten dürren Jahr wiederum bestätigte Eifahrung, daß richtig angelegte und gepflegte Futterflächen auf Moorboden einen unschätzbaren Rückhalt für die Futtergewinnung in niederschlag armen Jahren bieten.

Prof. Keppeler, Hannover, berichtet über „Den gegenwärtigen Stand der Torfrechnik". Trotz vieler Schwierigkeiten, insbesondere trotz der Zurückhallung der Verbraucher, bat sich die Torfindustrie im letzten Jahre weiter entwickelt und ihre Produktion von $2^{1 / 2}$ Millionen auf 3 Millionen Tonnen gesteigert. Die Herstellung des Torfes bat nur wenige Neuerungen zu verzeichnen, aber die Neuheiten des Vorjahres werden bei weiterer Ausbildung die so notwendige Verbilligung des Torfherstellungsprozesses bringen. Guter Torf hat sich als Brennstoff im Hausbrand, im Kleingewerbe und dem Dampfkessel der Industrie bestens bewährt. Besondere Beachtung fand er in Industrien, die den Torf vergasen (Glasindustrie und teilweise auch in der Eisenindustrie, wo seine reine Flamme sehr geschälzt wird). Wichtig ist der Übergang zum gebrochenen Torf ("NuBtorf"). Auch für die Pulverfeuerung sind neue Grundlagen geschaffen. Die Verkohlung des Torfes bringt guten Torfkoks und wertvolle Nebenprodukte hervor, aber die ausgedehnte Anwendung dieser Form der Torfverwendung stößt auf Schwierigkeiten. Mangels genügender Mengen an Nebenp rodukten ist es nicht möglich, sie ganz ihrem Charakter gemäß $\mathrm{zu}$ verarbeiten, vor allem aber hindert der hohe Torfpreis die umfassendere Anwendung dieser Formen, wie überhaupt die wichtigste Aufgabe für die Förderung der Torfindustrie die Vervollkommnung der Herstellungsinethode ist.

Oberingenieur Mickley, Vorsteher der technischen Abteilung des Vereins zur Förderung der Moorkultur im Deutschen Reiche, nahm Stellung zur ,Entwicklung der Torfmaschinen". im besonderen der Torfverarbeitungsmaschinen. Der Vortragende führte an Hand einer großen Anzahl von Lichtbildern die zahlreichen und vielseitigen Maschinenkonstruktionen vor Augen, die während einer langen Reihe von Jahren auf den einen Punkt gerichtet waren, die Beschaffenheit des 'Torfes zu verbessern. Mit Kinführung des Weberschen Verfahrens und der Dampfkraft und nach der unermüdlichen Arbeit einer großen Anzahl von Konstruktionsingenieuren gelang es, die Basis einer industriellen Torfgräberei zu finden und den Gedanken der Veredelung des Torfes durch maschinelle Bearbeitung bis zur heutigen Entwicklung durchzuführen.

Im Sonderausschulo für Spinnpflanzen sprach von Prittwit z, Kawallen, über , die gegenwärtige Lage des Flachsbaues und der Absatzverhältnisse für Flachs". Dabei wurde besonders darauf hingewiesen, daß die deutsche Leinenindustrie nur dann mit einem Anbau von Flachs in Deutschland in dem für sie notwendigen Maße würde rechnen können, wenn sie den Landwirten lohnende Preise für ihren Rohfỉachs zahlt. Es wurde als erstrebenswertes Ziel bezeichnet, die Landwirtschaft an den Gewinnen der Röstanstalten teilnehmen zu lassen, um auf dieser Grundlage die Gegensätze auszugleichen, die sich zwisthen den Flachsanbauern, den Röstanstalten und der Industrie zeigen.

Im Sonderausschuß für Forstdüngung wurde in der Diskussion des Vortrages von $S$ ü chtern, Hann.-Münden C.,Uber moderne Fragen der Forstkultur"), die Düngung der Waldböden mit Kalk, Kali und Stickstoff empfohlen.

Im Ausschuß der Düngerabteilung berichtete Lemmermann, Berlin, über „Kohlensäuredilngung".

v. Gerlach. Frankfurt a. $\mathrm{O}$ berichtete über die Phosphorsäurefrage". Während Böden, welche dauernd ohne Stalldünger bewirtschaftet werden, ein beträchtliches Phosphorsäurebedürfnis zeigen, ist dieses dort, wo innerhalb der Fruchtfolge ein oder mehrere Male mit Stallmist aboedüngt wird, im alloemeinen nicht hoch. Mit dem animalischen Dünger werden den Feldern große Mengen der durch die Ernten entzogenen Phosphorsäure wieder zugeführt, so daß derart behandelte Schläge den größten Teil der erforderlichen Phosphorsäure den Früchten liefern können. Infolgedessen sind die Ertragssteigerungen auf diesen Flärhen durch die Verwendung sonstiger phosphorsäurehaltiger Düngemittel nicht bedeutend und um so geringer, je stärker mit Superphosphat, Thomasmehl, Knochenmehlen usw. in den Vorjahran gedüngt worden ist. Es ist eine Anreicherung an Phosphorsäure eingetreten. Trotzdem wird man auch hier die Phosphorsäuredüngung selten mehrere Jahre hindurch vollständig unterlassen können. Dagegen ist es möglich, zu sparen. Der gegenwärtige Mangel an Phosphorsäure zwingt hierzu, und die hohen Preise für diesen Pflanzennähr toff lohnen dieses Verfahren.

Im Sonderausschuß zur Hehung des Lupinenbaues erläuterte Gerlach, Frankfurt a. 0 . in einem Vortrag ,Welche Wege sind einzuschlagen, um die Lupinenfrage auf breitere Basis zu stellen?" die Aufgaben des Sonderausschusses, die dahin zielen, durch eine großzügige Propaganda den Anbau von Samenlupinen in Deutschland orheblich zu steigern; ferner die Züchtung von Lupinen anzuregen, die 